\title{
PREDICTION OF LABOR ACTIVITY RECOGNITION IN CONSTRUCTION WITH MACHINE LEARNING ALGORITHMS
}

\author{
Ibrahim KARATAŞ \\ Osmaniye Korkut Ata University, Department of Civil Engineering, Osmaniye, Turkey
}

ORCID ID:

Abdulkadir BUDAK

Osmaniye Korkut Ata University, Department of Civil Engineering, Osmaniye, Turkey

\author{
ORCID ID:
}

\begin{abstract}
It is essential that the control and management of the work of labors in construction project management is effective. In this study, it is aimed to building artificial intelligence models to recognition on activities in a construction work to effectively utilization project management and control. In accordance with this purpose, 3axis accelerometer, gyroscope, and magnetometer data were obtained from the labors through the sensor to predict the activities determined for a construction work. These raw data were made compliance for the model by going through a series of preprocessing applications. These data are trained and modeled with basic machine learning algorithms logistic regression, SVC, DT and KNN algorithms. According to the results of the analysis, the best prediction was obtained with the SVC algorithm with an accuracy of $90 \%$. In other algorithms, respectively, $87 \%$ accuracy was contrived in the KNN algorithm, and approximately $80 \%$ accuracy in the logistic regression and DT algorithms. According to these values, it has been observed that the activities performed in a construction work can be estimated at a high rate. In this way, at the construction sites, it can be automatically determined which work the laborer do at a certain accuracy rate.
\end{abstract}

Keywords: Construction Management, Machine Learning, Activity Recognition, Labor

\section{INTRODUCTION}

Today's construction sector evolves and changes as a result of technological advancements. However, the construction industry's unique and complex structure prevents emerging technology from keeping up with this pace. Lately, research to adapt the construction industry to technology have been conducted. In this context, topics such as the BIM system, the Internet of Things, and Artificial Intelligence integration stand out (Akinosho et al., 2020; Babalola et al.; Oke et al., 2020; Xu et al., 2021).

Furthermore, because the construction industry is a labor-intensive sector, the impact of these technologies on labor becomes increasingly important (Calvetti et al., 2020). Therefore, much research has been done on the recognition of labor activities (Akhavian \& Behzadan, 2016; Al Jassmi et al., 2021; Antwi-Afari et al., 2019; Garcia-Gonzalez et al., 2020; Gondo \& Miura, 2020; Joshua \& Varghese, 2014; Ryu et al., 2016; Sanhudo et al., 2021; Sherafat et al., 2020). The automatic control of laborers' work is specifically addressed by labor activity recognition processes (Ryu et al., 2019). Sensors are used while laborers are working to accomplish this (Akhavian \& Behzadan, 2016). The most commonly used sensors are accelerometers and gyroscopes, which represent the acceleration and direction of movement of the human body (Alemayoh et al., 2021). Additionally, the measurement of the gravitational force received through the magnetometer sensor has also been stated to be useful in estimating labor activity recognition (Garcia-Gonzalez et al., 2020). Predicting the labor activities using these sensors has become increasingly significant in recent years. (Ryu et al., 2016) attempted to classify the laborer's activities using an accelerometer sensor placed on the laborer's wrist in his study. (Akhavian \& Behzadan, 2016), on the other hand, demonstrated that utilizing accelerometer and gyroscope data from a smartphone, it is possible to predict construction labor activity recognition. These predictions are made by creating artificial intelligence models. Logistic Regression (Akhavian \& Behzadan, 2016; Joshua \& Varghese, 2010; Ryu et al., 2019), Decision Tree (Akhavian \& Behzadan, 2016; Joshua \& Varghese, 2010; Martín et al., 2013; Ryu et al., 2019), Support Vector Machine (SVC) (Akhavian \& Behzadan, 2016; Kim et al., 2013; Sun et al., 2010), k Nearest Neighbors (KNN) (Akhavian \& Behzadan, 2016; Ryu et al., 2019), Neural Network (Akhavian \& Behzadan, 2016), and Naive Bayes (Joshua \& Varghese, 2010; Martín et al., 2013) are some of the models used in labor activity recognition prediction.

Received 4 August 2021; Received in revised form 25 August 20Z1; Accepted 20 September 2021;

Available online 25 September 2021;

doi: 10.4629I//CLNTECHvol5iss3pp38-47 
There is not enough research on the use of artificial intelligence in the construction industry, as well as on labor activity recognition prediction, according to the literature reviewed in this study. In addition, one of the sensors used for labor activity recognition, the accelerometer sensor was either used alone or combined with a gyroscope. As a result, in this study, accelerometer, gyroscope, and magnetometer data were combined to undertake labor activity recognition prediction research. In addition, logistic regression, SVC, KNN, and decision tree techniques were used to analyze the data. As a result of these analyses, it was determined which model higher accurately predicted laborer activities.

\section{MATERIALS AND METHODS}

In this study, it is aimed to estimate the activities of laborers in a construction industry. In this direction, the artificial intelligence model designed to predict a laborer's activities in the context of a soil test setup in a laboratory is implemented step by step, according to the flowchart shown in the Figure 1.

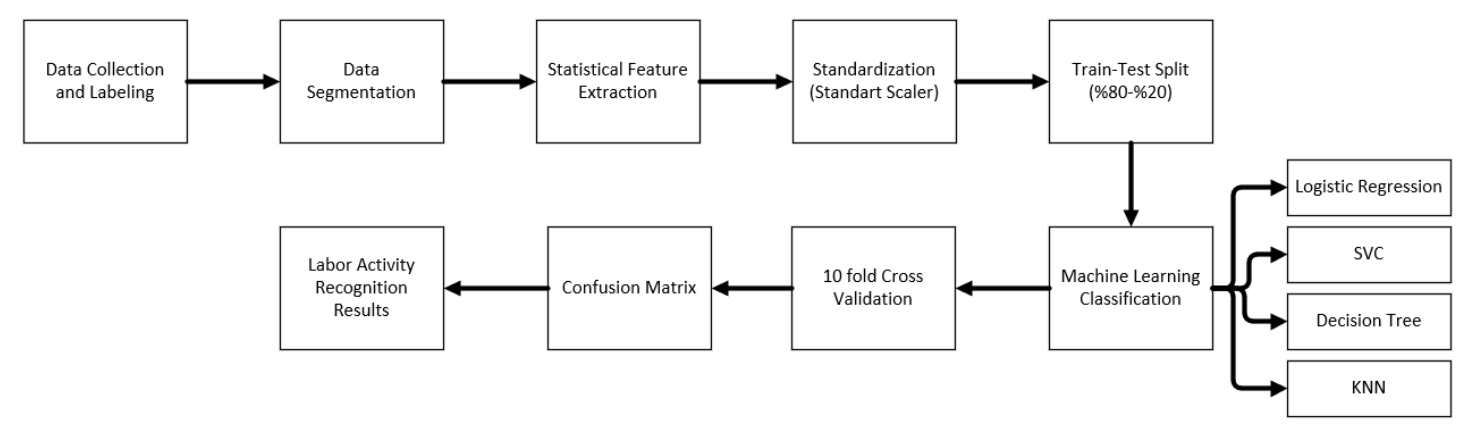

Figure 1. Flowchart for labor activity recognition prediction

First, 3-axis accelerometer, gyroscope, and magnetometer data were obtained with the help of the sensor attached to the laborer's arm as in the Figure 2. These obtained data were labeled to predict logging, carrying, surfacing, vibrating, and waiting activities for a soil test setup created in the laboratory environment. A total of 76080 data were obtained from the activities performed for approximately 25 minutes. The distribution of these data appeared as shown in the Figure 3. As an example of these activities, the visualization of the logging activity's accelerometer, gyroscope and magnetometer data in $\mathrm{x}, \mathrm{y}$, and $\mathrm{z}$ directions is shown in the Figure 4.

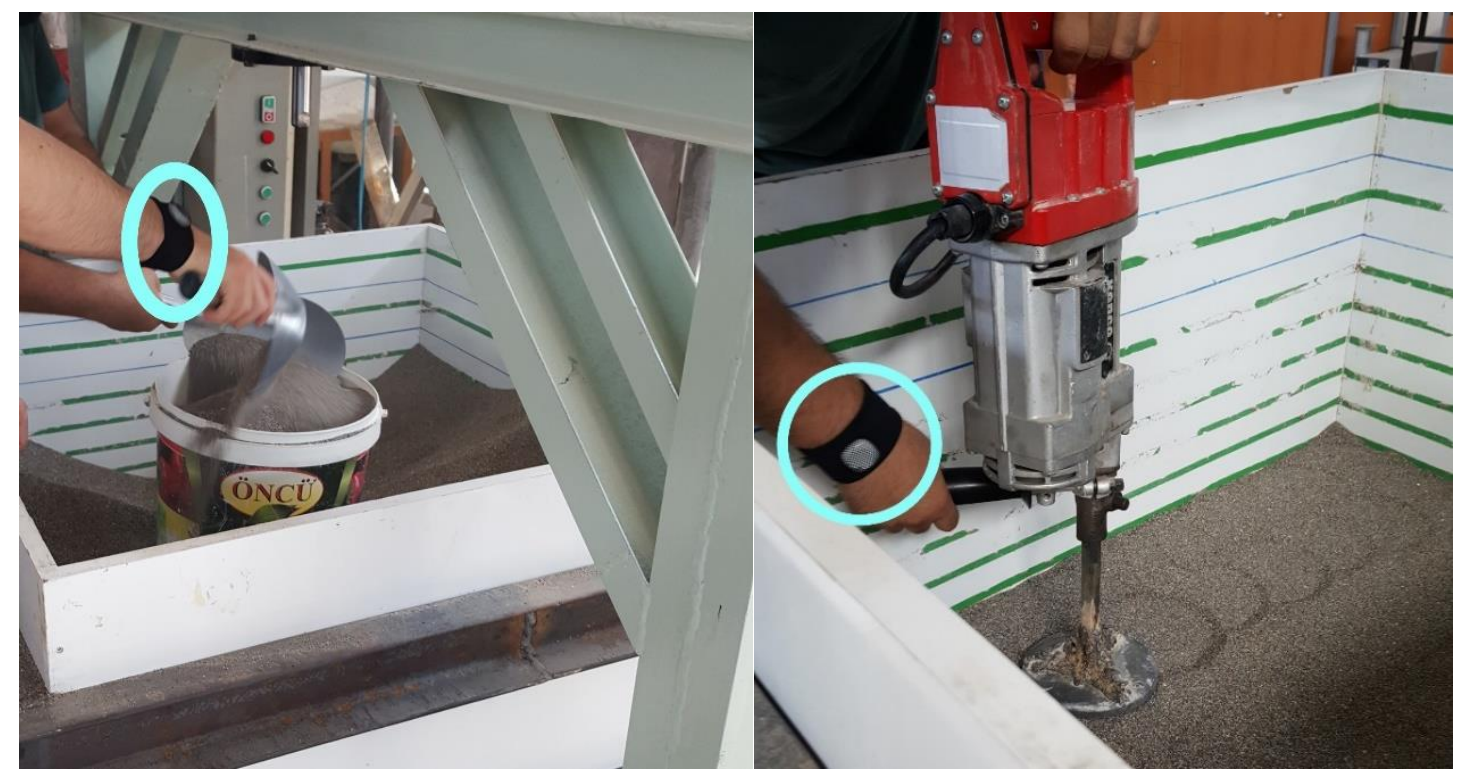

Figure 2. Data collection with sensor attached to the laborer's arm

Received 4 August 2021; Received in revised form 25 August 20Z1; Accepted 20 September 2021;

Available online 25 September 2021;

dai: 10.46291//[CDNTECHvo|Siss3pp380-47 


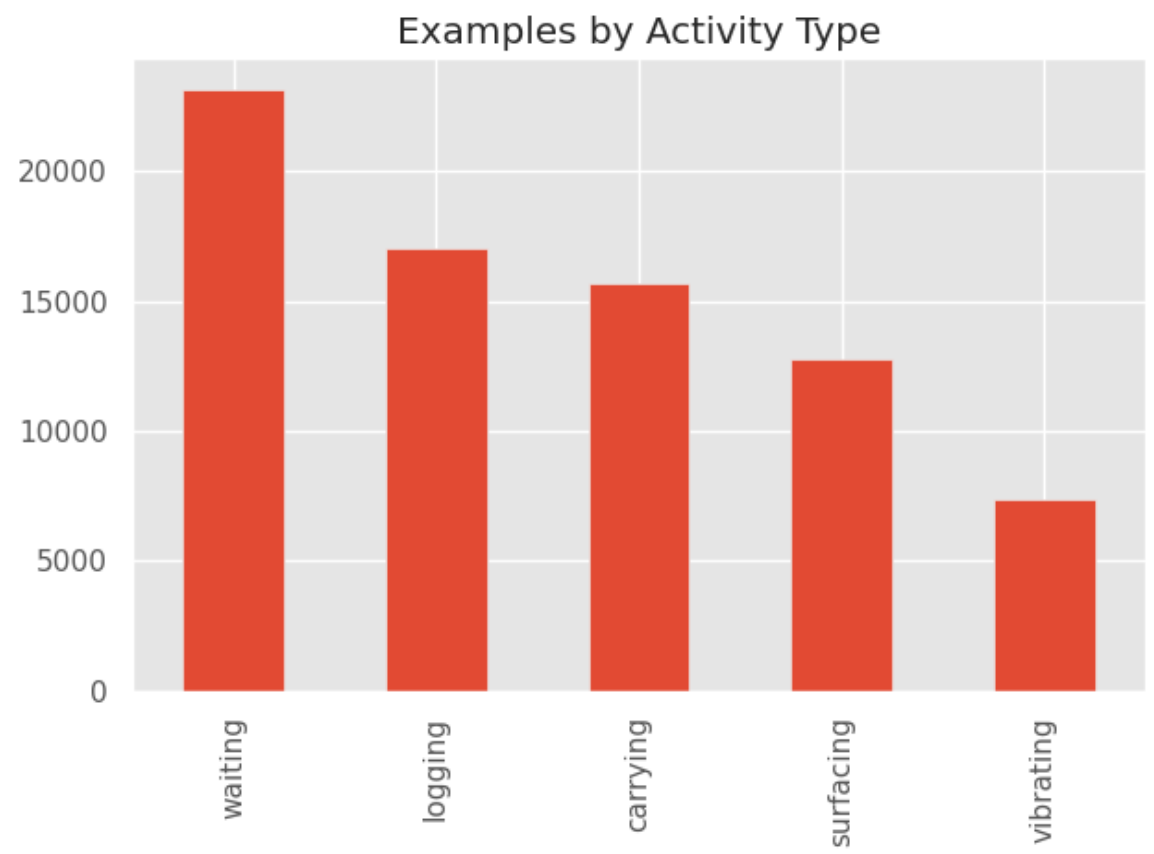

Figure 3. Activity-based distribution of obtained data
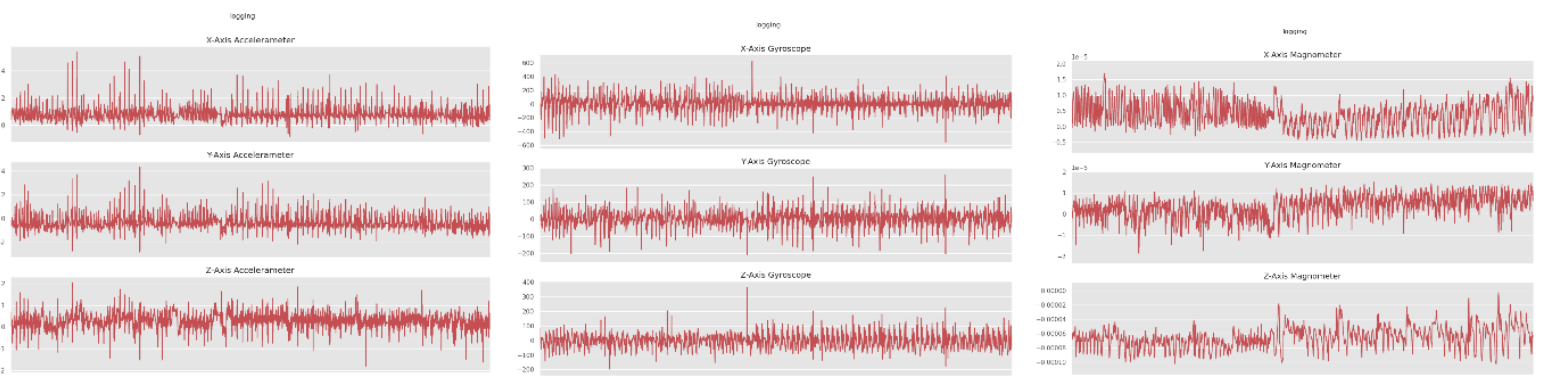

Figure 4. 3-axis sensor data from logging activity

Then, data segmentation was applied to make this data suitable for the model. With this application, the data is separated into fixed width sliding windows consisting of 60 data, starting from the beginning. These windows are created by running on top of each other with $50 \%$ overlap. After this process, statistical feature extraction was performed on each window. The features calculated for each axis of the accelerometer, gyroscope and magnetometer data for each window are given in the Table 1. A total of 81 feature extractions were made with 9 statistical features calculated.

Table 1. Features and description created with statistical feature extraction

\begin{tabular}{ll}
\hline Statistical Features Names & Description \\
\hline Sum values & Calculates the sum over the seperated windows values \\
\hline Median & Calculates the median the seperated windows values \\
\hline Mean & Calculates the mean the seperated windows values \\
\hline Length & Calculates the length the seperated windows values \\
\hline Standard deviation & Calculates the standard deviation the seperated windows values \\
\hline Variance & Calculates the variance the seperated windows values \\
\hline Root mean score & Calculates the quadratic mean the seperated windows values \\
\hline Maximum & Calculates the maximum value the seperated windows values \\
\hline
\end{tabular}

Received 4 August 20Z1; Received in revised form 25 August 20Z1; Accepted 20 September 2021;

Available online 25 September 2021;

doi: I0.4629I//CDNTECHvol5iss3pp38-47 
After statistical feature extraction, it is aimed to create a more accurate model by standardizing the data with a mean value of 0 and a standard deviation of 1 . Dependent variables (y) coded by labeling the standardized independent variables (x); It is divided into two as train and test at a rate of $80 \%-20 \%$. In this way, the accuracy of the model will be calculated by comparing with the test data, the values obtained as a result of the model created with the train data. In this study, 4 basic machine learning classifier algorithms, namely logistic regression, support vector classifier, decision tree classifier and $\mathrm{k}$ nearest neighbor classifier, were used for the prediction of labor activities.

Following the creation of the machine learning models, k-fold cross validation was used to verify whether the models were random and to eliminate the data's bias (Farooq et al., 2021). In this study, the recommended 10-fold cross validation (Kohavi, 1995) was performed to obtain the optimum variance. With 10-fold cross validation, the data is divided into 10 layers, and in each turn, 1 different floor validation data and the other 9 layers are analyzed as train data. CV accuracy values were calculated by taking the average of the accuracy values found for each floor.

The constructed models were evaluated and compared using four different assessment metrics: accuracy score, precision, recall, and f1-score. The equations provided below are used to calculate these evaluation metrics.

$$
\begin{aligned}
& \text { Accuracy }=\frac{T P+T N}{T P+T N+F P+F N} \\
& \text { Precision }=\frac{T P}{T P+F P} \\
& \text { Recall }=\frac{T P}{T P+F N} \\
& F 1 \text { score }=2 * \frac{\text { Precision } * \text { Recall }}{\text { Precision }+ \text { Recall }}
\end{aligned}
$$

TP (True Positives) and TN (True Negatives) values from the values given in Equations 1, 2, and 3 show the correctly predicted values of the model. FN (False Negatives) and FP (False Positives) values are expressions that show the incorrectly predicted values of the model. Accuracy score expresses the ratio of correctly predicted values to all values. The Precision score shows how many of the values we predicted as positive were positive. This metric is especially important when the cost of the FP predict value is high. Recall, on the other hand, shows how many of the transactions we need to predict positively are positively predicted. This metric is helpful when the cost of the FN predict value is high. F1 score, on the other hand, shows a result that allows us to ignore the extreme cases by taking the harmonic average of the precision and recall values. It is also important because it is a measurement metric that includes all values, not just FN and FP values.

\section{RESULT AND DISCUSSION}

The performance of the models was evaluated in this study by using basic machine learning algorithms to analyze data from the sensor for automatic recognition of construction activities. It is feasible to evaluate the predict performance of each model using the confusion matrix created as a result of these analyses.

The normalized confusion matrix obtained as a result of the analyzes made in the logistic regression model is as shown in the Figure 5. Accordingly, the activity with the highest prediction success rate of 95\% in this model is surfacing activity. Although the logging, vibrating, and waiting activities have a relatively high prediction rate, they did not show a high enough prediction success. Carrying activity, on the other hand, had the lowest predicting performance among the activities with a $57 \%$ predicting success. 


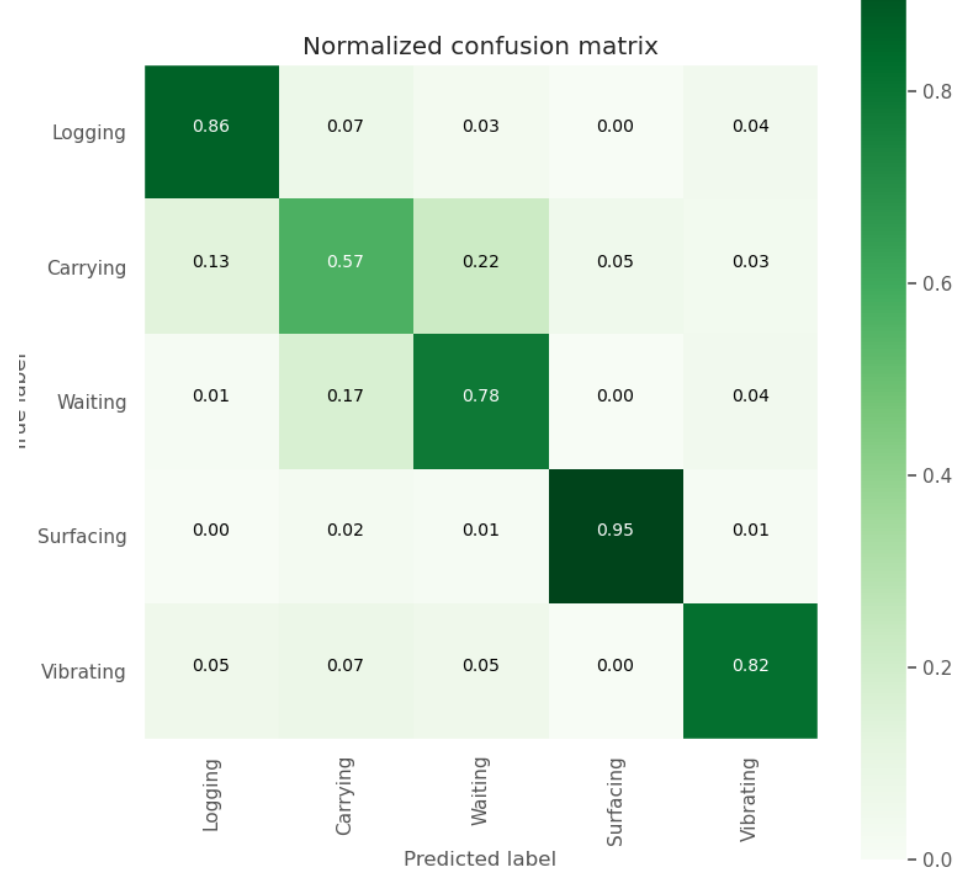

Figure 5. Logistic regression model confusion matrix

The normalized confusion matrix obtained as a result of the analyzes made in the support vector classifier (SVC) model is as shown in the Figure 6. Accordingly, the activity with the highest prediction success rate of $98 \%$ in this model is surfacing activity. Logging, vibrating, and waiting activities can be said to have a relatively high prediction rate. However, although carrying activity had a relatively high rate of prediction with $88 \%$, it was the activity with the lowest predicting performance among labor activities.

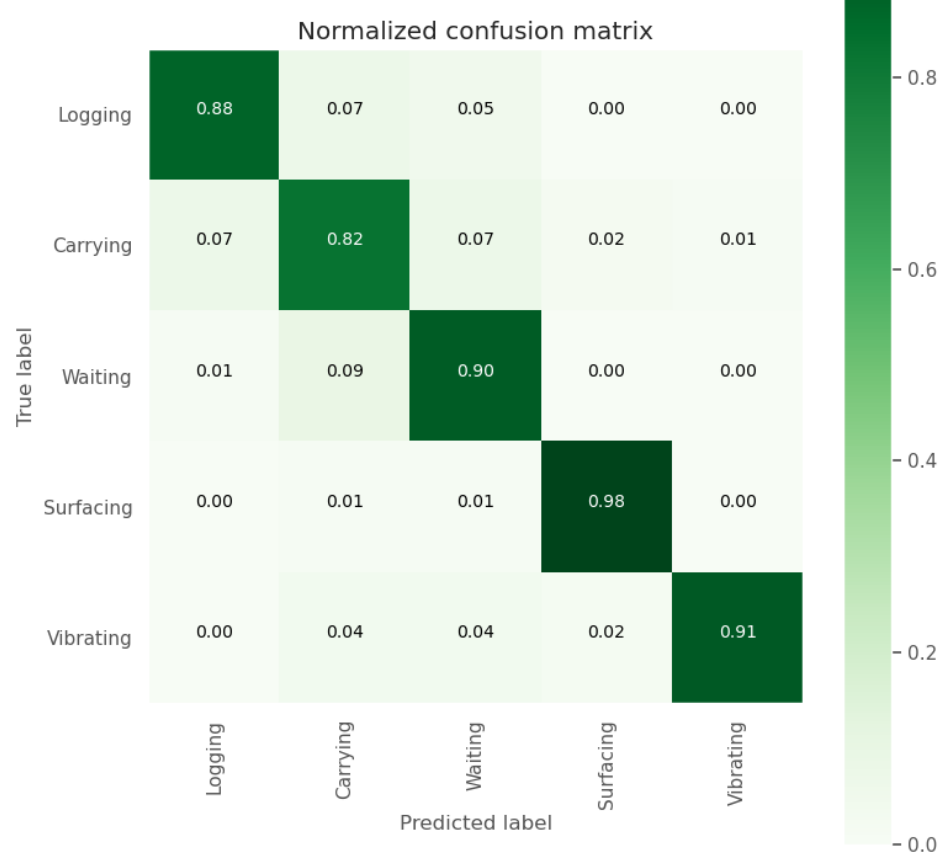

Figure 6. Support vector classifier (SVC) model confusion matrix

Received 4 August 2021; Received in revised form 25 August 2021; Accepted 20 September 2021;

Available online 25 September 2021;

dai: 10.4629I//CDNTECHvolSiss3pp38-47 
The normalized confusion matrix obtained as a result of the analyzes made in the Decision Tree (DT) classifier model is as shown in the Figure 7. Accordingly, the activity with the highest prediction success rate of $91 \%$ in this model is surfacing activity. Although the logging, vibrating, and waiting activities have a relatively high prediction rate, they did not show a high enough prediction success. Carrying activity, on the other hand, had the lowest predicting performance among the activities, with a forecasting success of $65 \%$.

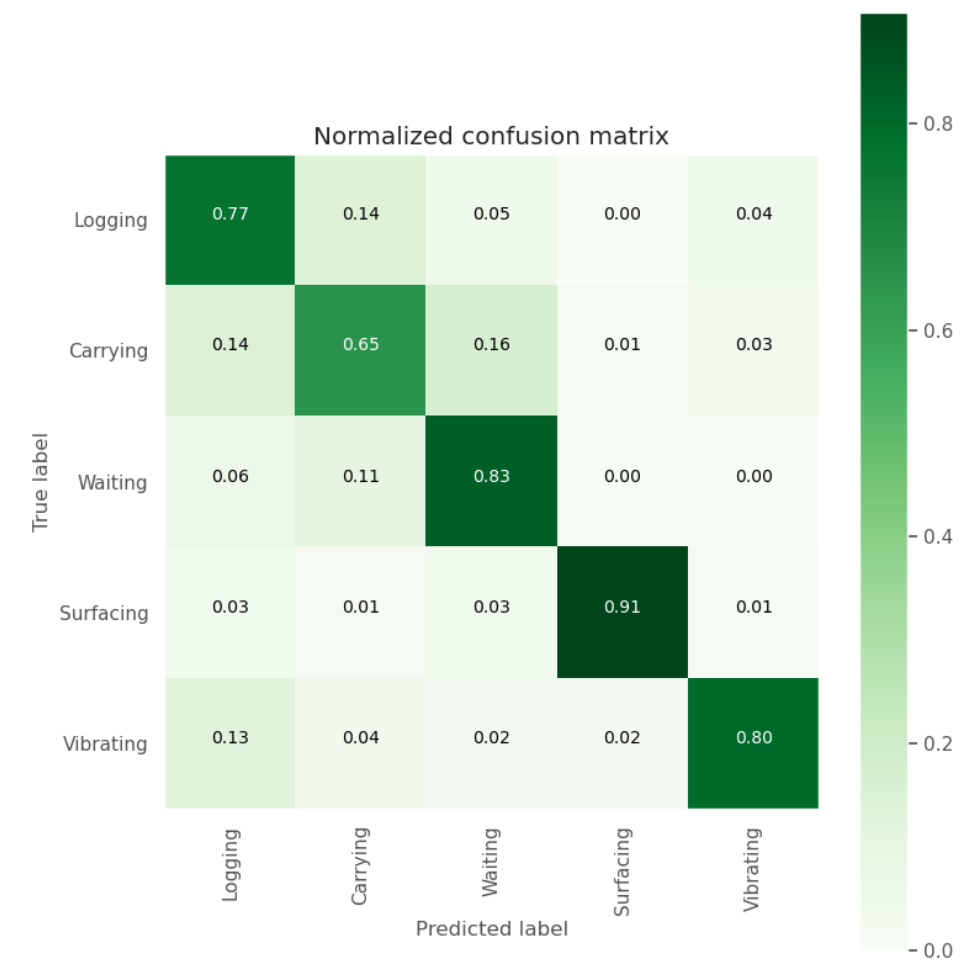

Figure 7. Decision Tree (DT) classifier model confusion matrix

The normalized confusion matrix obtained as a result of the analyzes made in the K Nearest Neighbor (KNN) classifier model is as shown in the Figure 8. Accordingly, the activity with the highest prediction success rate of $98 \%$ in this model seems to be surfacing and vibrating activities. Logging and waiting activities can be considered as relatively high predictive. However, in carrying activity, it was the activity with the lowest prediction performance among the activities with a prediction success rate of $74 \%$. 


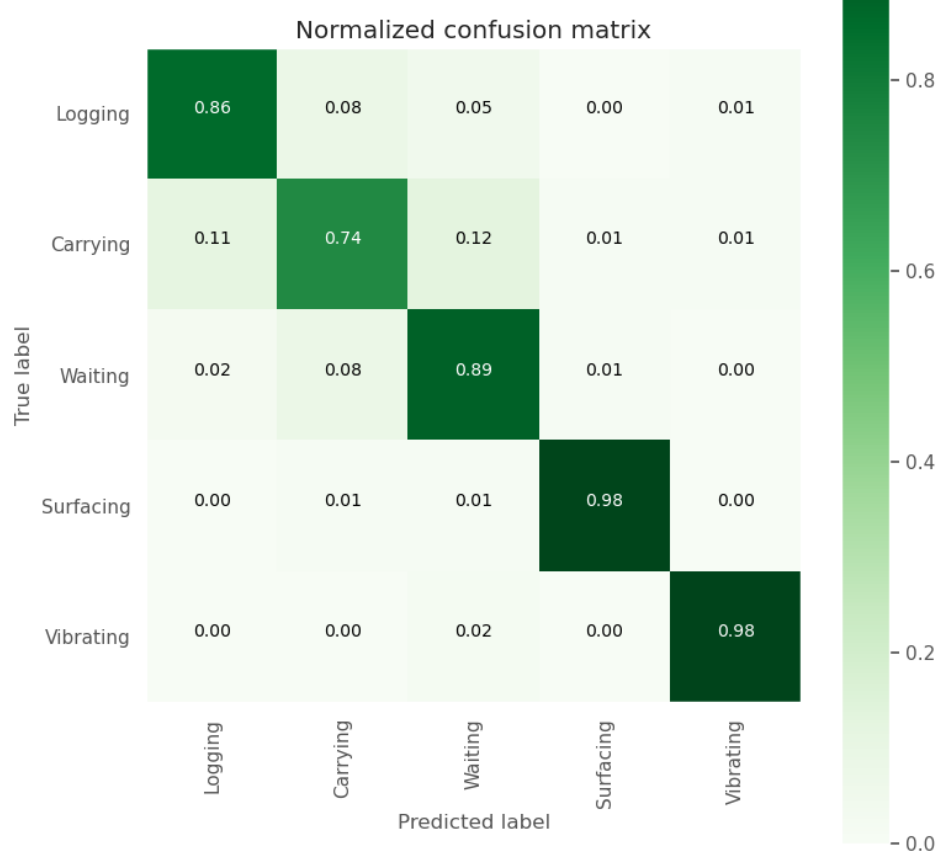

Figure 8. K nearest neighbors (KNN) model confusion matrix

As a result of the evaluations made according to the confusion matrix, it is seen that the surfacing activity is predicted more accurately in all models and the carrying and waiting activities is generally predicted less accurately than other activities. This is because surfacing and waiting activities have similar movements. On the other hand, as shown in the Table 2, precision, recall and f1-score values were calculated and evaluated for each activity in each model. Accordingly, it can be said that the SVC algorithm generally has higher values than other algorithms in most activities.

Table 2. Evaluation metrics by algorithms and activities

\begin{tabular}{clccc}
\hline $\begin{array}{c}\text { Machine Learning } \\
\text { Models }\end{array}$ & Activities & Precision & Recall & Fl-Score \\
\hline \multirow{3}{*}{ Logistic Regression } & Logging & 0.84 & 0.86 & 0.85 \\
\cline { 2 - 5 } & Carrying & 0.65 & 0.57 & 0.60 \\
\cline { 2 - 5 } & Waiting & 0.74 & 0.78 & 0.76 \\
\cline { 2 - 5 } & Surfacing & 0.94 & 0.95 & 0.95 \\
\cline { 2 - 5 } SVC & Vibrating & 0.79 & 0.82 & 0.80 \\
\cline { 2 - 5 } & Logging & 0.92 & 0.88 & 0.90 \\
\cline { 2 - 5 } & Carrying & 0.81 & 0.82 & 0.82 \\
\hline \multirow{2}{*}{ Waiting } & 0.86 & 0.90 & 0.88 \\
& Surfacing & 0.97 & 0.98 & 0.97 \\
\cline { 2 - 5 } Decision Tree & Vibrating & 0.98 & 0.91 & 0.94 \\
& Logging & 0.72 & 0.77 & 0.67 \\
\cline { 2 - 5 } & Carrying & 0.69 & 0.65 & 0.80 \\
\cline { 2 - 5 } & Waiting & 0.77 & 0.83 & \\
\hline
\end{tabular}

Received 4 August 2021; Received in revised form 25 August 2021; Accepted 20 September 20Z1; 
Journal homepage: http://icontechjournal.com/index.php/iij

Volume 5 (2021) Issue 3

\begin{tabular}{lllll}
\hline & Surfacing & 0.97 & 0.91 & 0.94 \\
\cline { 2 - 5 } Vibrating & 0.85 & 0.80 & 0.82 \\
\hline \multirow{2}{*}{$K N N$} & Logging & 0.87 & 0.86 & 0.87 \\
\cline { 2 - 5 } & Carrying & 0.81 & 0.74 & 0.77 \\
\cline { 2 - 5 } & Waiting & 0.83 & 0.89 & 0.86 \\
\cline { 2 - 5 } & Surfacing & 0.98 & 0.98 & 0.98 \\
\cline { 2 - 5 } & Vibrating & 0.96 & 0.98 & 0.97 \\
\hline
\end{tabular}

The accuracy scores of the models are explained in two ways in this study. Each model was assessed first by training accuracy scores, and then by accuracy values generated by employing 10-fold CV, which is a more accurate and robust technique to model evaluation. Accordingly, training and 10-fold CV accuracy scores of logistic regressions, SVC, Decision tree and KNN algorithms are shown in the Table 3. As a result, the SVC algorithm's accuracy values are higher than those of other methods, and the 10-fold CV value appears to be increasing. After the SVC algorithm, the algorithm with the highest accuracy seems to be the KNN algorithm. Decision tree and Logistic regression algorithms have lower accuracy values compared to other algorithms.

4. Table 3. Accuracy score of machine learning models

\begin{tabular}{lcccc}
\hline & Logistic Regression & SVC & Decision Tree & KNN \\
\hline Training & 0.79 & 0.89 & 0.79 & 0.88 \\
10-fold CV & 0.81 & 0.90 & 0.78 & 0.87 \\
\hline
\end{tabular}

\section{CONCLUSION}

Despite the rapid development of technology around the world, technology in the construction industry is progressing very slowly. As a result, there are insufficient academic research on the application of technology in construction management. Since the construction sector is a labor-intensive sector, in this study, it is aimed to predict the recognition of laborers' activities with artificial intelligence in line with the data obtained from construction laborers. As a result, it is anticipated that the automatic estimation of laborer activities and the controls performed by technical employees at construction sites would be automated, and construction activity will be facilitated.

Within the scope of this study, artificial intelligence prediction models were created from basic machine learning algorithms for the recognition of labor activities for placing sand in a test box in a soil test setup experiment established in a laboratory environment. It was investigated which model outperformed the models created with Logistic regression, SVC, Decision tree and KNN algorithms. Accordingly, it has been determined that the SVC algorithm performs better than other algorithms. With this algorithm, it is seen that the algorithms made by the laborer are estimated correctly at $90 \%$. In the KNN algorithm, it is predicted correctly at a rate of $87 \%$, but it has a relatively low estimation ability with $80 \%$ accuracy values of other algorithms.

The results obtained from the analyses are thought to be sufficient. Using advanced alternative models, on the other hand, can result in better prediction performance. Deep learning applications based on artificial neural networks are also predicted to improve prediction accuracy. As a result of the sensors attached to the labor, the tracking of the labor and the tasks they perform may be regulated automatically, and an efficient working strategy can be followed. Furthermore, the use of technology in construction management is supposed to help with the three building milestones of time, cost, and quality.

\section{REFERENCES}

Akhavian, R., \& Behzadan, A. H. (2016). Smartphone-based construction workers' activity recognition and classification. Automation in Construction, 71, 198-209. https://doi.org/10.1016/j.autcon.2016.08.015

Received 4 August 20Z1; Received in revised form 25 August 2021; Accepted 20 September 2021;

Available online 25 September 2021;

dai: 10.4629I//CLNTECHvo|Siss3pp38-47 
Akinosho, T. D., Oyedele, L. O., Bilal, M., Ajayi, A. O., Delgado, M. D., Akinade, O. O., \& Ahmed, A. A. (2020). Deep learning in the construction industry: A review of present status and future innovations. Journal of Building Engineering, 32, 101827. https://doi.org/10.1016/j.jobe.2020.101827

Al Jassmi, H., Al Ahmad, M., \& Ahmed Soha (2021). Automatic recognition of labor activity: A machine learning approach to capture activity physiological patterns using wearable sensors. Construction Innovation.

Alemayoh, T. T., Lee, J. H., \& Okamoto, S. (2021). New sensor data structuring for deeper feature extraction in human activity recognition. Sensors, 21(8), 2814. https://doi.org/10.3390/s21082814

Antwi-Afari, M. F., Li, H., Seo, J., \& Wong, A. Y. L. (Eds.) (2019). Automated Recognition of Construction Workers' Activities for Productivity Measurement Using Wearable Insole Pressure System.

Babalola, A., Musa, S., Akinlolu, M. T., \& Haupt, T. C. A bibliometric review of advances in building information modeling (bim) research. Journal of Engineering, Design and Technology. https://www.emerald.com/insight/content/doi/10.1108/JEDT-01-2021-0013/full/pdfUR https://www.emerald.com/insight/content/doi/10.1108/JEDT-01-2021-0013/full/html

Calvetti, D., Mêda, P., Chichorro Gonçalves, M., \& Sousa, H. (2020). Worker 4.0: The future of sensored construction sites. Buildings, 10(10), 169. https://doi.org/10.3390/buildings10100169

Farooq, F., Ahmed, W., Akbar, A., Aslam, F., \& Alyousef, R. (2021). Predictive modeling for sustainable highperformance concrete from industrial wastes: A comparison and optimization of models using ensemble learners. Journal of Cleaner Production, 292, 126032. https://doi.org/10.1016/j.jclepro.2021.126032

Garcia-Gonzalez, D., Rivero, D., Fernandez-Blanco, E., \& Luaces, M. R. (2020). A public domain dataset for reallife human activity recognition using smartphone sensors. Sensors (Basel, Switzerland), 20(8). https://doi.org/10.3390/s20082200

Gondo, T., \& Miura, R. (2020). Accelerometer-based activity recognition of workers at construction sites. Frontiers in Built Environment, 6, Article 563353. https://doi.org/10.3389/fbuil.2020.563353

Joshua, L., \& Varghese, K. (Eds.) (2010). Construction Activity Classification Using Accelerometers. American Society of Civil Engineers.

Joshua, L., \& Varghese, K. (2014). Automated recognition of construction labour activity using accelerometers in field situations. International Journal of Productivity and Performance Management, 63(7), 841-862. https://doi.org/10.1108/IJPPM-05-2013-0099

Kim, T.-S., Cho, J.-H., \& Kim, J. T. (2013). Mobile motion sensor-based human activity recognition and energy expenditure estimation in building environments. Sustainability in Energy and Buildings, 987-993. https://doi.org/10.1007/978-3-642-36645-1_87

Kohavi, R. (Ed.) (1995). A Study of Cross-Validation and Bootstrap for Accuracy Estimation and Model Selection.

Martín, H., Bernardos, A. M., Iglesias, J., \& Casar, J. R. (2013). Activity logging using lightweight classification techniques in mobile devices. Personal and Ubiquitous Computing, 17(4), 675-695. https://doi.org/10.1007/s00779-012-0515-4

Oke, A. E., Arowoiya, V. A., \& Akomolafe, O. T. (2020). Influence of the internet of things' application on construction project performance. International Journal of Construction Management, 1-11. https://doi.org/10.1080/15623599.2020.1807731

Ryu, J., Seo, J., Jebelli, H., \& Lee, S. (2019). Automated action recognition using an accelerometer-embedded wristband-type activity tracker. Journal of Construction Engineering and Management, 145(1), 4018114. https://doi.org/10.1061/(ASCE)CO.1943-7862.0001579

Ryu, J., Seo, J., Liu, M., Lee, S., \& Haas, C. T. (Eds.) (2016). Action Recognition Using a Wristband-Type Activity Tracker: Case Study of Masonry Work.

Sanhudo, L., Calvetti, D., Martins, J. P., Ramos, N. M., Mêda, P., Gonçalves, M. C., \& Sousa, H. (2021). Activity classification using accelerometers and machine learning for complex construction worker activities. Journal of Building Engineering, 35, 102001. https://doi.org/10.1016/j.jobe.2020.102001

Sherafat, B., Ahn, C. R., Akhavian, R., Behzadan, A. H., Golparvar-Fard, M., Kim, H., Lee, Y.-C., Rashidi, A., \& Azar, E. R. (2020). Automated methods for activity recognition of construction workers and equipment: State-of-the-art review. Journal of Construction Engineering and Management, 146(6), 3120002. https://doi.org/10.1061/(ASCE)CO.1943-7862.0001843

Received 4 August 20Z1; Received in revised form 25 August 20Z1; Accepted 20 September 2021;

Available online 25 September 2021;

doi: 10.4629I//CDNTECHvol5iss3pp38-47 
Sun, L., Zhang, D., Li, B., Guo, B., \& Li, S. (Eds.) (2010). Activity Recognition on an Accelerometer Embedded Mobile Phone with Varying Positions and Orientations. https://link.springer.com/chapter/10.1007/978-3642-16355-5_42

Xu, Y., Zhou, Y., Sekula, P., \& Ding, L. (2021). Machine learning in construction: From shallow to deep learning. Developments in the Built Environment, 6, 100045. https://doi.org/10.1016/j.dibe.2021.100045 